\title{
TITLE:
}

\section{Chaos and Period-Doubling Bifurcations in a Simple Acoustic System}

\section{$\operatorname{AUTHOR}(\mathrm{S})$ :}

Kitano, M.; Yabuzaki, T.; Ogawa, T.

\section{CITATION:}

Kitano, M. ... [et al]. Chaos and Period-Doubling Bifurcations in a Simple Acoustic System. Physical Review Letters 1983, 50(10): 713-716

ISSUE DATE:

1983-03

URL:

http://hdl.handle.net/2433/152439

RIGHT:

c 1983 The American Physical Society 


\title{
Chaos and Period-Doubling Bifurcations in a Simple Acoustic System
}

\author{
M. Kitano, T. Yabuzaki, and T. Ogawa \\ Radio Atmospheric Science Center, Kyoto University, Uji 611, Japan
}

(Received 16 August 1982)

\begin{abstract}
Chaotic oscillations due to delay-induced instabilities are easily seen with a simple and familiar acoustic system. The measured bifurcation diagram to chaos, which is very different from that for a one-dimensional map model, is compared with numerical results. The bifurcation structure is dominated by the ratio of the response time to the delay time of the system.
\end{abstract}

PACS numbers: $05.40 .+\mathrm{j}, 42.65 .-\mathrm{k}, 43.90 .+\mathrm{v}$

The chaotic or turbulent behavior seen in a physical system which is governed by deterministic equations has attracted intense interest recently. Ikeda, Daido, and Akimoto ${ }^{1}$ have pointed out that chaotic behavior can occur in an optically bistable system which is described by a differential-difference equation. Experimentally, Gibbs et al. ${ }^{2}$ have observed such optical turbulence or Ikeda instability in an optical hybrid device with a delay in the feedback. In a recent article ${ }^{3}$ they showed that their system takes the period-doubling route to chaos. It is surprising that the period-doubling scenario seems to be realized in many different physical systems. ${ }^{3,4}$

In this paper we report the observation of an Ikeda-type instability and novel period-doubling bifurcations in a simple acoustic system composed of a microphone, a nonlinear circuit, an amplifier, and a speaker. The experimental setup is shown in Fig. 1 . The time delay $t_{k}$ which plays a key role in inducing instabilities corresponds to the propagation time of sound from the speaker to the microphone which are faced about $13 \mathrm{~cm}$ apart $\left(t_{R} \sim 0.37 \mathrm{~ms}\right)$. The other key element is a nonlinear circuit which has at least one peak in its input versus output characteristic curve. The most popular and easily constructed circuit which has a peak is a full-wave rectifier.

We could hear chaotic oscillation when the amplifier gain was high enough, whereas without the rectifier only periodic oscillations could be observed. In the following experiment to observe the period-doubling bifurcations we used a rectifier with operational amplifiers ${ }^{5}$ which has more precise characteristics than the conventional twodiode rectifier shown in Fig. 1. The output $V_{y}$ and the input $V_{x}$ are related by the equation $V_{y}$ $=-\left|V_{x}+V_{x_{0}}\right|+V_{y 0}$, where $V_{x_{0}}$ and $V_{y_{0}}$ are the input and output offset voltages, respectively. As described later, an adjustment of the offsets is needed to observe the period-doubling bifurcations.
By the analogy of our system to those in Refs. $1-3$, we introduce the differential-difference equation

$$
\gamma^{-1} \dot{x}(t)=-x(t)+\mu F_{1}\left(x\left(t-t_{R}\right)\right),
$$

with

$$
F_{1}(x)=-|x+0.5|+0.5,
$$

where $x=V / 2 V_{0}, V$ is the voltage fed to the speaker, $V_{0}$ is the input offset of the rectifier, and $\mu$ is the loop gain. The response time $\gamma^{-1}$ of the amplifier was set at about $0.15 \mathrm{~ms}$.

In the experiment we set $V_{y 0}=V_{x 0}$ so that the condition $F_{1}(0)=0$ is satisfied, which assures that $x=0$ is a equilibrium point. The small-amplitude oscillation is expected to be almost symmetric with respect to the equilibrium point and to have a small dc component. Thus we can neglect the effect that the dc component cannot pass through the feedback loop in the actual system.

Equation (1) has a nonlinearity $F_{1}$ with a sharp peak whereas the equations treated by Ikeda, Daido, and Akimoto ${ }^{1}$ and Gibbs et al..$^{2,3}$ have round smooth peaks which are approximated by a quadratic function. In the theory of one-dimensional maps, these two types of functions may be viewed as representatives. ${ }^{6}$

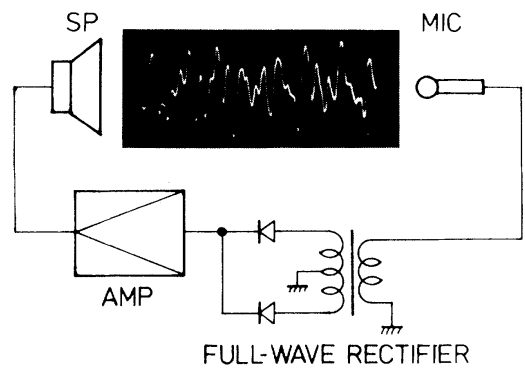

FIG. 1. Experimental setup. A microphone (MIC), a full-wave rectifier, an amplifier (AMP), and a speaker (SP) form a feedback loop. An example of the chaoticsound wave form is also shown. 
Our system shows various modes of oscillation, such as periodic oscillation with period $\sim 2\left(t_{R}\right.$ $\left.+\gamma^{-1}\right)$, oscillations with much smaller period, oscillations modulated with long period ( $10 \mathrm{~ms})$, chaotic oscillation, or intermittent chaotic oscillation, some of which are not expected from Eq. (1). Perhaps this is because we have neglected in Eq. (1) the low-frequency response of the system, phase shifts of the speaker and the microphone, and the room acoustics. The appearance of each mode depends in a complicated manner on parameters such as the amplifier gain or the position of the microphone. However, near threshold we could observe the period-doubling bifurcations to chaos with good reproducibility.

We show an example of such bifurcations in Fig. 2. As the amplifier gain is increased, periodic oscillation [Fig. 2(a)] begins, which we may call "period-two" oscillation, ${ }^{3}$ for its period is about $2\left(t_{R}+\gamma^{-1}\right)$. Next the period doubling to period four [Fig. 2(b)] occurs. The bifurcations to period-eight [Fig. 2(c)] and to chaotic oscillations [Fig. 2(d)] follow successively.

Sometimes in the course of the bifurcations, low-frequency oscillation $(\sim 100 \mathrm{~Hz})$ begins to be superimposed and the bifurcation series is interrupted. Such a low-frequency instability can be removed by decreasing the low-frequency gain of the amplifier.

Figure 3 shows the bifurcation diagram obtained experimentally. The horizontal axis of a cathode-ray tube (CRT) is swept by the ramp voltage applied to the voltage-controlled amplifier (VCA) which is inserted in the feedback loop to vary the parameter $\mu$ slowly. The output of the microphone is applied to the vertical axis.
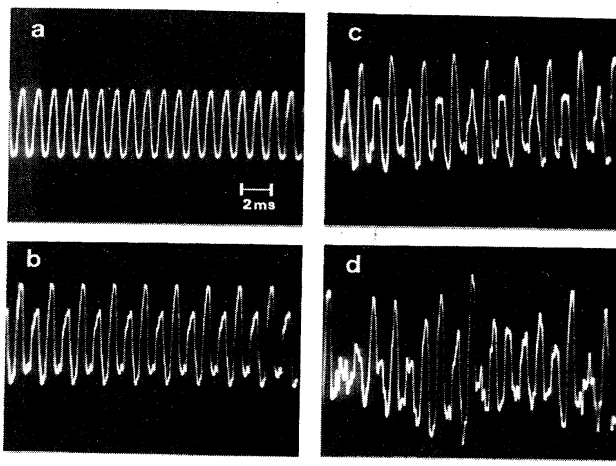

FIG. 2. The output voltage of the microphone. As the amplifier gain increased, (a) period-two, (b) periodfour, (c) period-eight, and (d) chaotic oscillations appear successively.
The horizontal trace on the left means that no oscillation takes place for small values of $\mu$. Next we see the period-two oscillation build suddenly up to a level determined by the offset of the rectifier. The top peaks and the bottom peaks of the period-two wave form [Fig. 2(a)] are seen as bright edges, whose separation corresponds to the amplitude of the oscillation. The enhancement of the edges takes place because the vertically oscillating beam spot of the CRT moves slowly there.

As $\mu$ is increased one finds that each edge splits into two branches which correspond to the four principal peaks of the period-four wave form in Fig. 2(b). The innermost two excess branches due to the subpeaks are also seen. The interval of the period-eight oscillation is too narrow to be observed.

Next there comes the chaotic region which can hardly be seen in Fig. 3(a) for no enhancement on the CRT occurs. By increasing the beam intensity we can see the chaotic region [Fig. 3(b)].

Let us return to Eq. (1). In the limit $t_{R} \gamma \gg 1$, namely when the time response of the system is extremely fast, Eq. (1) is reduced to a difference equation or a one-dimensional map $^{1}$ :

$$
x_{n+1}=\mu F_{1}\left(x_{n}\right) \text {. }
$$

It is well known that when $F_{1}$ is replaced by a quadratic function such as $F_{2}=-x(x+1)$, the bifurcation diagram shows a series of pitchfork bifurcations at $\mu=\mu_{k}$ with period doubling by $2^{k}$, $k=1,2, \ldots{ }^{7}$ There is an accumulation point $\mu_{\infty}$ to which $\left\{\mu_{k}\right\}$ converges, above which the chaotic
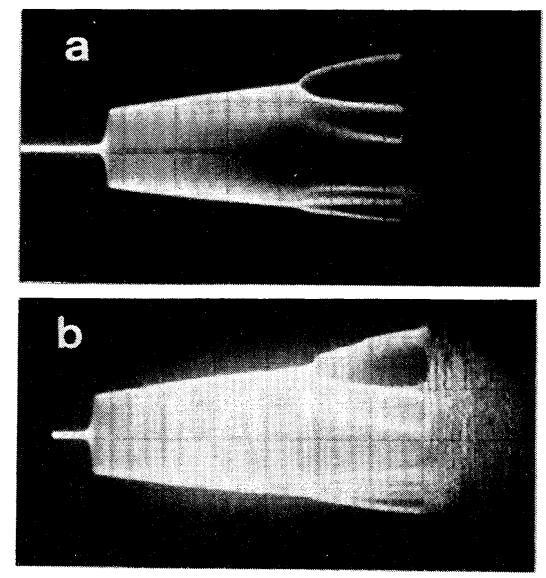

FIG. 3. (a) Bifurcation diagram, i.e., output voltage vs loop gain $\mu$ which is swept by VCA. (b) Same as (a) except the beam intensity of the CRT is increased to see the chaotic region. 
behavior appears. This is a route to chaos seen in various physical systems. Another feature seen in the diagram is band mergings or inverse bifurcation of the chaotic bands. As $\mu$ is increased, the chaotic bands merge in pairs successively until fully developed chaos appears. Schematically the bifurcations can be summarized as follows: $P_{0} \rightarrow P_{1} \rightarrow \cdots \rightarrow$ (onset of chaos) $\rightarrow \cdots$ $\rightarrow P_{(1)} \rightarrow P_{(0)}$, where $P_{k}$ and $P_{(k)}$ represent the region of period $2^{k}$ and that of period- $2^{k}$ chaos, ${ }^{3}$ respectively.

For the map $F_{1}$, which contains the absolute value function, the bifurcation diagram is quite different. In Fig. 4(a), we have plotted the iterative values $x_{n}$ of Eq. (3) for each $\mu$. We can see the bifurcations $P_{0} \rightarrow$ (onset of chaos) $\rightarrow \cdots \rightarrow P_{(1)}$ $\rightarrow P_{(0)}$. Namely, the bifurcation points $\mu_{k}(k$ $=1,2, \ldots)$ are degenerate at the point $\mu=1$. Thus period-doubling bifurcations cannot be seen and chaotic oscillation begins suddenly. The period- doubling bifurcations, however, are observed experimentally in our system in spite of the nonlinearity $F_{1}$. Perhaps this is because the condition $t_{R} \gamma \gg 1$ which reduces Eq. (1) to Eq. (3) is not satisfied in our case.

We solved Eq. (1) numerically to see the effect of finite response time $\gamma^{-1}$ on the bifurcation diagram. The diagrams in Figs. 4(b)-4(d) were obtained as follows. For each $\mu$, we calculated the stationary solution $x(t)$ of Eq. (1) during $50 t_{R}$. Then we picked times $t_{p}$ where $\dot{x}\left(t_{p}\right)=0$ (dot denotes $d / d t)$ and plotted the values $x\left(t_{p}\right)$. Although, as in the diagram obtained experimentally, there appear spurious branches due to subpeaks in $x(t)$, we can see how the bifurcations proceed as $\mu$ was increased.

In the case of $t_{R} \gamma=9.0$, the diagram [Fig. 4(b)] is fairly similar to Fig. 4(a) except for the portion just after the first bifurcation. The periodtwo region $\left(P_{1}\right)$ appears. The width of the upper
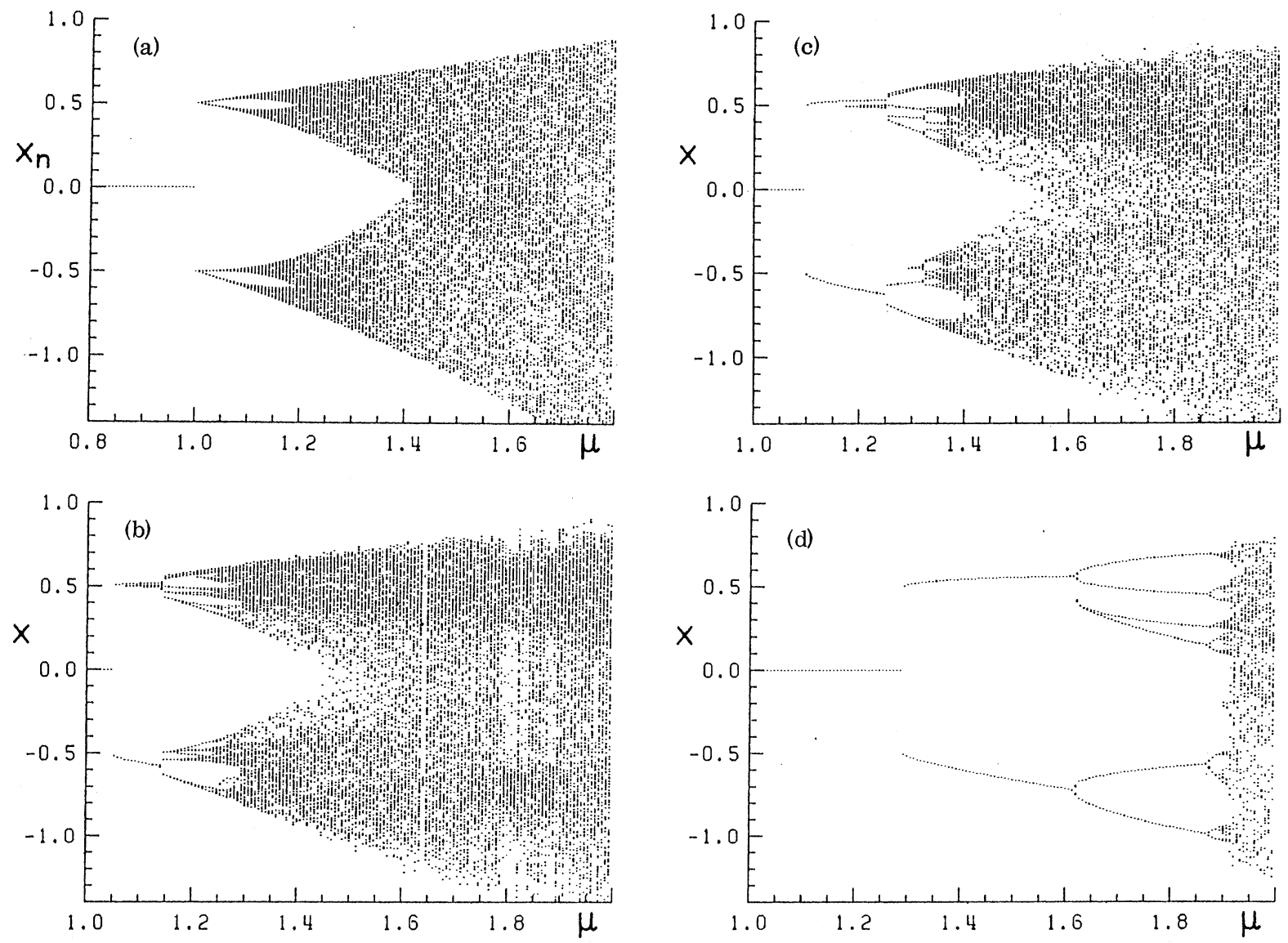

FIG. 4. (a) Bifurcation diagram for the difference equation (3): 150 successive plots of $x_{n}$ after preiteration for each $\mu$. Bifurcation diagrams for the differential-difference equation (1) with (b) $t_{R} \gamma=9.0$, (c) $t_{R} \gamma=6.0$, and (d) $t_{R} \gamma$ $=3.0$. The figures are obtained by plotting the peak values of the stationary solution during $50 t_{R}$ for each $\mu$. 
branch comes from the subpeaks of the wave form, not from the chaotic behavior. Above the second bifurcation we can see some band mergings of the chaotic oscillation as in Fig. 4(a).

It is interesting to note that the chaotic regime is changed to the ordered regime by the effect of $\gamma{ }^{8}$ The newly appearing region may be $P_{(1)}$ not $P_{1}$. The discrimination between them by numerical methods is very difficult but there is reason to believe that it is $P_{1}$ as described later.

By increasing $t_{R} \gamma$, we see that the $P_{1}$ region extends and the transition to chaos is delayed. We also see the bifurcation to $P_{2}$ and that to $P_{3}$ [Fig. 4(c)]. We note that Fig. 4(d) for $t_{R} \gamma=3$ is qualitatively similar to the diagram obtained in our experiment where $t_{R} \gamma$ is estimated to be $\sim 2.5$.

The period-doubling bifurcations seen in Fig. 4(d) convince us that the newly appearing region is $P_{k}(k=1,2,3)$ rather than $P_{(k)}$ because the latter bifurcate inversely as $\mu$ is increased. Another interesting feature in Fig. 4 is that as $t_{R} \gamma$ is decreased the periodic regions $\left(P_{k}\right)$ extend at the expense of the chaotic regions $\left(P_{(k)}\right)$. In Fig. 4(a) we see only $P_{0}$ as a periodic region, whereas in Fig. 4(d) there seems to be only $P_{(0)}$ as a chaotic region.

In summary, we have observed the Ikeda-type instability in a simple acoustic system. The system bifurcates to chaos through period doublings. The numerical analysis explains well the novel bifurcation diagram observed experimentally and shows that the bifurcation structure is sensitive to the time response of the system. One of the matters to be clarified is the detailed structure near the onset of chaos, for example, whether the bifurcation series is truncated or not, and if not, what is the value of the Feigenbaum constant. We wish to thank Y. Ueda for helpful discussions. This work was supported in part by the Ministry of Education, Science and Culture in Japan, under a Grant-in-Aid for Scientific Research. One of us (M.K.) is a recipient of the Sakkokai Foundation Fellowship.

${ }^{1}$ K. Ikeda, H. Daido, and O. Akimoto, Phys. Rev. Lett. 45, 709 (1980).

${ }^{2}$ H. M. Gibbs, F. A. Hopf, D. L. Kaplan, and R. L. Shoemaker, Phys. Rev. Lett. 46, 474 (1981).

${ }^{3}$ F. A. Hopf, D. L. Kaplan, H. M. Gibbs, and R. L. Shoemaker, Phys. Rev. A 25, 2172 (1982).

${ }^{4} \mathrm{~J}$. Testa, J. Perez, and C. Jeffries, Phys. Rev. Lett. 48, 714 (1982); J. Maurer and A. Libchaber, J. Phys. (Paris), Lett. 40, 419 (1979); M. Giglio, S. Mus-

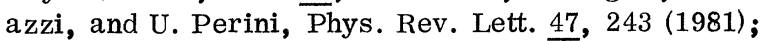
J. M. Wersinger, J. M. Finn, and E. Ott, Phys. Rev. Lett. 44, 453 (1980).

${ }^{5} \mathrm{See}$, for example, J. G. Graeme, Applications of Operational Amplifiers (McGraw-Hill, New York, 1973).

${ }^{6}$ E. Ott, Rev. Mod. Phys. 53, 655 (1981).

${ }^{7}$ P. Collet and J. P. Eckmann, Iterated Maps on the Interval as Dynamical Systems (Birkhauser, Boston, 1980).

${ }^{8}$ Another mechanism for disappearance of the chaotic region has been found and discussed by C. Grebogi, E. Ott, and J. A. Yorke, Phys. Rev. Lett. 48,1507 (1982). 


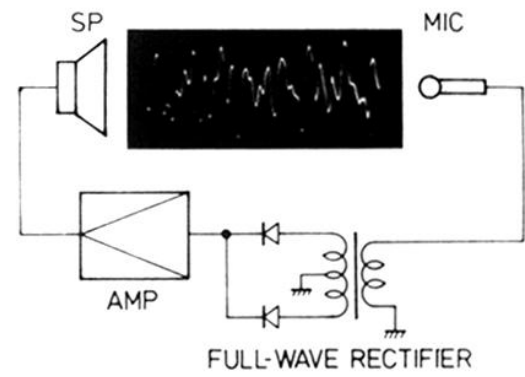

FIG. 1. Experimental setup. A microphone (MIC), a full-wave rectifier, an amplifier (AMP), and a speaker (SP) form a feedback loop. An example of the chaoticsound wave form is also shown. 

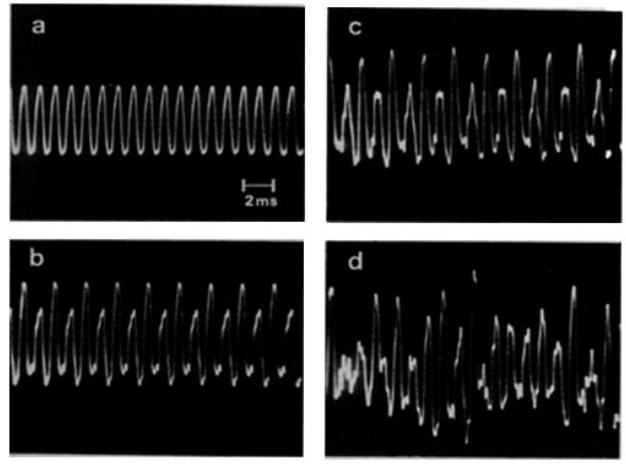

FIG. 2. The output voltage of the microphone. As the amplifier gain increased, (a) period-two, (b) periodfour, (c) period-eight, and (d) chaotic oscillations appear successively. 

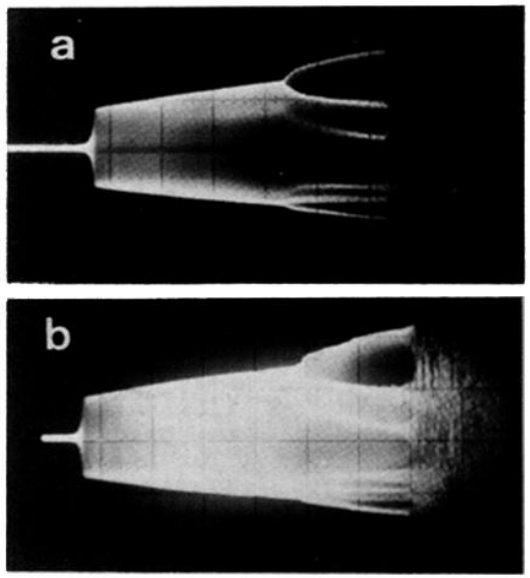

FIG. 3. (a) Bifurcation diagram, i.e., output voltage vs loop gain $\mu$ which is swept by VCA. (b) Same as (a) except the beam intensity of the CRT is increased to see the chaotic region. 